$\mathbb{P}$ periodica polytechnica

Civil Engineering

58/4 (2014) 335 338

doi: $10.3311 /$ PPci.7638

http://periodicapolytechnica.org/ci

Creative Commons Attribution (1)

RESEARCH ARTICLE

\section{Three-layer in-plane dynamical track model for metro applications}

\author{
Vilmos Zoller / István Zobory
}

Received 2013-12-23, revised 2014-03-09, accepted 2014-06-17

\begin{abstract}
In the study a linear dynamical model suitable for metroapplications is constructed and analysed in the framework of the Winkler-foundation theory. The metro track is allocated within the inner space of the tube of big diameter. The tube is considered as a beam on elastic and damped foundation. The track itself consists also of two elastically supported beams, namely the basement beam made of steel-reinforced concrete is situated at the bottom of the tube and connected to the lower-inner surface of the tube by an elastic and dissipative "carpet-pad". The concrete beam supports the rails through an elastic and dissipative layer. The wheel loads acting on the rails are moving horizontally along the track. The set of partial differential equations describing the vertical motion of the three-layer dynamical system is composed and the method of solution in the case of travelling wheel loads is elaborated.
\end{abstract}

\section{Keywords}

railway track model $\cdot$ distributed parameter system $\cdot$ vehicle dynamics $\cdot$ motion equations $\cdot$ motion responses

\section{Vilmos Zoller}

Sándor Rejtő Faculty of Light Industry and Environmental Protection Engineering, Óbuda University, Doberdó út 6, H-1034 Budapest, Hungary e-mail: zoller.vilmos@rkk.uni-obuda.hu

\section{István Zobory}

Department of Railway Vehicles and Vehicle System Analysis, Faculty of Transportation Engineering and Vehicle Engineering, Budapest University of Technology and Economics,

Múegyetem rkp. 3, H-1111 Budapest, Hungary

e-mail: zobory@rave.vjt.bme.hu

\section{Introduction}

The investigation into the dynamics of metro tracks is important, first of all from the point of view of dimensioning the load bearing structures of the track. The metro trains follow each other at a very high frequency. The moving loads cause dynamical load-variations that lead to cumulative damage of fatigue in the structural components and (sometimes irregular) wear process on the railheads. On the other hand, the different geometrical rail irregularities and the inhomogenities in the rail supporting stiffness cause excited vibrations of the vehicles, giving rise again to component fatigue and reduction in running comfort. A third very important sphere of problems is the noise and vibration generation caused by the metro traffic in the tube. The dynamical analysis can respond the questions concerning the advantageous track constructions and materials to be applied. In this paper a general purpose linear track/vehicle model is introduced for metro applications.

\section{The three-layer model}

In Fig. 1 the structure of the "deep-led" metro track is plotted. The tube containing the track in strict sense, is laid in the tunnel bored into the soil, and is supported by the resultant vertical distributed soil-pressure. The tube can be represented in this sense as a beam on approximately linear Winkler's foundation. Within the tube mentioned, a longitudinally extended basement (this long continuous concrete beam is sometimes called "tread") made of steel-reinforced concrete is allocated. The connection of the concrete basement beam and the inner bottom surface of the tube is realised by a "carpet-pad" having approximately linear vertical stiffness and damping. The pair of rails supporting the metro vehicles are connected with the concrete basement beam through continuous linearly elastic and dissipative supporting spots, which can also be modelled approximately by a continuous Winkler's foundation. Thus, the elastically/dissipative connected three beams is called a three-layer model. In the Figure the three beams as three layers in question can easily be recognised. The metro vehicle is rolling on the rails along the axis of the tube. The vertical connection of the supporting rails with the wheel-sets is realised by the linearized 
vertical Hertzian springs and dampers. The metro vehicle itself is modelled by a finite dimension linear system. In the vertical in-plane track model the vertical displacement of the rails (two rails working in parallel) from the unloaded equilibrium position is designated by $z_{1}(x, t)$, the vertical displacement of the concrete basement from the unloaded equilibrium position is designated by $z_{2}(x, t)$, and finally the vertical displacement tube from its unloaded equilibrium position is $z_{3}(x, t)$. The three bivariate functions introduced above are the unknown function-triple, describing the motion state of the metro track by an appropriately composed set of partial differential equations.

As a possible simplified alternative metro track model version, consider the structure plotted in Fig. 2 The simple twolayer model consists of the longitudinally extended and elastically supported concrete basement, undergoing vertical displacement $z_{2}(x, t)$ relative to its unloaded equilibrium position, and of the elastically supported pair of rails, which perform vertical displacement $z_{1}(x, t)$ also relative to its unloaded equilibrium position. The metro vehicle moving along the track model is treated similarly as it has been described above in the framework of the three-layer model.

\section{Mathematical models}

Our mathematical modelling system consists of the following three simultaneous partial differential equations:

$$
\begin{aligned}
& B_{1} z_{1}=f-S_{1}\left(z_{1}-z_{2}\right), \\
& B_{2} z_{2}=S_{1}\left(z_{1}-z_{2}\right)-S_{2}\left(z_{2}-z_{3}\right), \\
& B_{3} z_{3}=S_{2}\left(z_{2}-z_{3}\right)-S_{3} z_{3},
\end{aligned}
$$

representing the track/vehicle system of a metro system. The vehicles move on the elastically supported rails, which are laying on the elastically supported concrete tread, embedded elastically into a metro tube. The vertical wheel-tread forces mean longitudinally moving load on the "upper layer", namely on the rails. In our investigation it is enough to consider a single horizontally moving force $f$, represented by a travelling phasor. For treating the interaction of the track with the whole vehicle (4 wheel-sets) the principle of superposition can be applied due to the linearity of the track/vehicle dynamical model used.

The partial differential equations above are governing the motion of the three idealized Bernoulli-Euler beams, i. e. that of the rail, the concrete and the tube, respectively, where fourth-order partial differential operators $B_{i}$ and first-order partial differential operators $S_{i}$ are defined by relations

$$
\begin{aligned}
& B_{i}:=I_{i} E_{i} \partial_{x}^{4}+\rho_{i} A_{i} \partial_{t}^{2}, \\
& S_{i}:=k_{i} \partial_{t}+s_{i},
\end{aligned}
$$

representing the beams and their supports, respectively.

Here $I_{i}, E_{i}, \rho_{i}$ and $A_{i}$ are the usual parameters of the corresponding Bernoulli-Euler beams. Furthermore $s_{i}$ and $k_{i}$ are the stiffness and damping coefficients of the underlying visco-elastic Winkler supports, respectively, for integers $i=$ $1,2,3$. The units of measure of the latters are $\left[s_{i}\right]=\mathrm{N} / \mathrm{m}^{2}$ and $\left[k_{i}\right]=\mathrm{Ns} / \mathrm{m}^{2}$.

The damped oscillatory load is moving along the rail at a constant velocity $v$, and the loading force acting along the rail has the form

$$
f:=f_{0} e^{w t} \delta(x-v t), w \in C,
$$

with $\delta$ standing for Dirac's $\delta$-distribution.

Symbols $f_{0}$ and $w$ above denote the amplitude and the complex frequency of the loading force, respectively, with the latter moving along the rail at a constant velocity $v$ corresponding to beam operator $B_{1}$.

The system of partial differential equations has to satisfy three boundary conditions:

$$
\lim _{x \rightarrow \pm \infty} z_{1}(x, t)=\lim _{x \rightarrow \pm \infty} z_{2}(x, t)=\lim _{x \rightarrow \pm \infty} z_{3}(x, t)=0 .
$$

A rearrangement of the partial differential equations above results in a new form of our partial differential equations as

$$
\begin{aligned}
& D_{1} z_{1}=f+S_{1} z_{2}, \\
& D_{2} z_{2}=S_{1} z_{1}+S_{2} z_{3}, \\
& D_{3} z_{3}=S_{2} z_{2},
\end{aligned}
$$

where the fourth-order partial differential operators on the left-hand side are defined by relations

$$
\begin{aligned}
& D_{1}:=B_{1}+S_{1}, \\
& D_{2}:=B_{2}+S_{1}+S_{2}, \\
& D_{3}:=B_{3}+S_{2}+S_{3} .
\end{aligned}
$$

From the system of partial differential equations above one can conclude the relations

$$
\begin{aligned}
& D_{1} D_{2} D_{3} z_{1}= \\
& =D_{2} D_{3} f+S_{1} D_{3}\left(S_{1} z_{1}+S_{2} z_{3}\right)= \\
& =D_{2} D_{3} f+S_{1}^{2} D_{3} z_{1}+S_{1} S_{2}^{2} z_{2}= \\
& =D_{2} D_{3} f+S_{1}^{2} D_{3} z_{1}+S_{2}^{2}\left(D_{1} z_{1}-f\right)= \\
& =\left(D_{2} D_{3}-S_{2}^{2}\right) f+\left(S_{1}^{2} D_{3}+S_{2}^{2} D_{1}\right) z_{1},
\end{aligned}
$$

and, finally, a partial differential equation of order 12

$$
\left(D D_{1}-S_{1}^{2} D_{3}\right) z_{1}=D f
$$

can be obtained with the help of the introduction of a new 8th-order partial differential operator $D$, defined by formula

$$
D:=D_{2} D_{3}-S_{2}^{2} \text {. }
$$

Excitation $D f$ on the right-hand side of partial differential equation

$$
\left(D D_{1}-S_{1}^{2} D_{3}\right) z_{1}=D f
$$




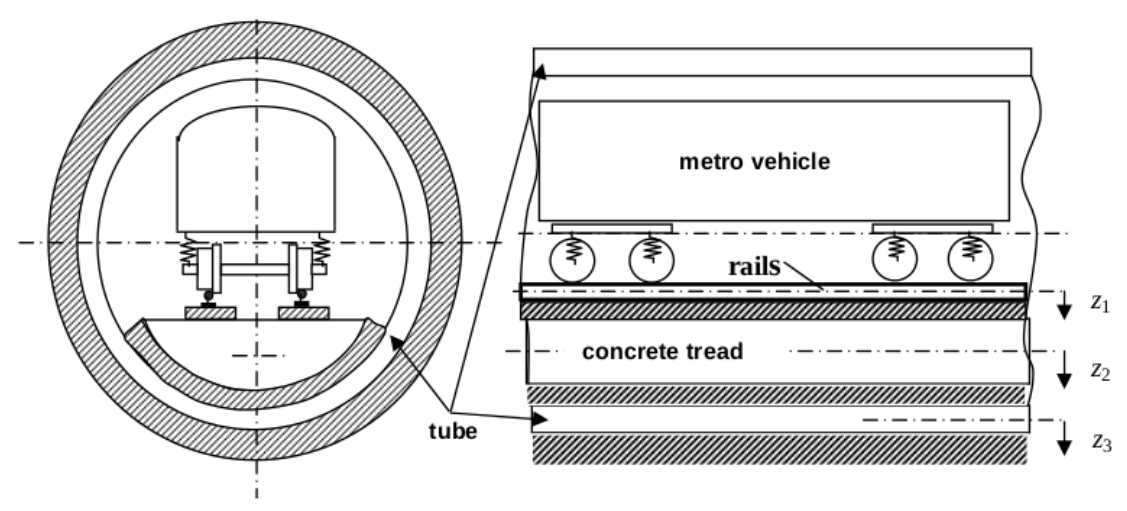

Fig. 1. Three-layer model with three beams

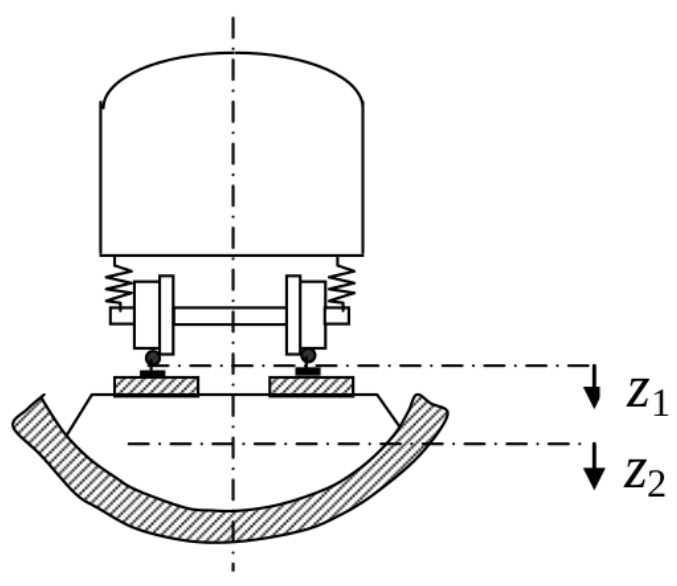

Fig. 2. Two-layer model with two beams

is a linear combination of the derivatives of Dirac's $\delta$ distribution, multiplied by a phasor factor $e^{w t}$.

\section{Solution of the system equations}

We are looking for the solution to the problem in the usual form [1,2]:

$$
z_{1}(x, t)=e^{w t} u(\xi),
$$

introducing relative horizontal displacement $\xi:=x-v t$ as a new independent variable instead of longitudinal variable $x$.

Applying the "Ansatz" (test function) above, we conclude to a linear ordinary differential equation of form

$$
\sum_{i=0}^{12} a_{i} u^{(i)}=\sum_{i=0}^{8} c_{j} \delta^{(j)},
$$

with constant coefficients, which has a particular solution in the form of a linear combination as

$$
u(\xi)=\sum_{i=0}^{8} c_{j} U^{(j)}(\xi) H(\xi) .
$$

Here function $U$ stands for the solution to the corresponding homogeneous equation

$$
\sum_{i=0}^{12} a_{i} u^{(i)}=\sum_{i=0}^{8} c_{j} \delta^{(j)},
$$

satisfying initial conditions for $U$ and its derivatives as follows:

$$
\begin{aligned}
& U^{(j)}(0)=0, \\
& U^{X I}(0)=1 / a_{12} .
\end{aligned}
$$

Characteristic equation

$$
P(\lambda)=\sum_{i=0}^{12} a_{i} \lambda^{i}=0
$$

of the ordinary differential equation above has 12 roots, where (under certain conditions) the first six roots $\lambda_{1}, \lambda_{2}, \ldots, \lambda_{6}$ are placed in the left half-plane of the complex plane $C$, while the next six roots $\lambda_{7}, \lambda_{8}, \ldots, \lambda_{12}$ lay in the right half-plane.

The investigation of the critical frequencies is still an open problem.

This way, cf. [1-4], one is able to satisfy the boundary condition corresponding to variable $z_{1}$, and obtain solution function $z_{1}$ in the form

$$
\begin{aligned}
& z_{1}(x, t)= \\
& =\sum_{i=1}^{12} \frac{\sigma_{i}}{P^{\prime}\left(\lambda_{i}\right)} \sum_{j=0}^{8} c_{j} \lambda_{i}^{j} e^{w t+\lambda_{i}(x-v t)} H\left(\sigma_{i}(x-v t)\right),
\end{aligned}
$$

where $\sigma_{i}=-\operatorname{sgn}\left(\operatorname{Re}\left(\lambda_{i}\right)\right)$ holds. 
Symbol $P^{\prime}$, in the above solution function $z_{1}$, stands for the derivative of characteristic polynomial $P$ of the ordinary differential equation

$$
\sum_{i=0}^{12} a_{i} u^{(i)}=\sum_{i=0}^{8} c_{j} \delta^{(j)},
$$

while $H$ denotes Heaviside's unit jump function.

The other two solution functions $z_{2}(x, t)$ and $z_{3}(x, t)$ can be given by the consecutive solution of the following two first-order ordinary differential equation problem:

$$
\begin{aligned}
& S_{1 z_{2}}=D_{1} z_{1}-f, \\
& S_{2} z_{3}=D_{2} z_{2}-S_{1} z_{1},
\end{aligned}
$$

by taking the boundary conditions corresponding to variables $z_{2}$ and $z_{3}$, respectively, into consideration.

\section{Conclusions}

In the study of the dynamics of the three-layer track we have dealt with three coupled beam equations on Winkler's foundations, one of them loaded by a vertical phasor excitation moving longitudinally at a constant travelling velocity. The solution to the above problem is elaborated in the paper on the basis of a certain operator analysis and by using a proper moving phasor "Ansatz". Using the roots of the deduced characteristic equation and Heaviside's unit jump function we are able to express the displacement of the rails (the upper layer) as a response to the moving excitation in a closed-form fashion. For the determination of the displacement functions for the medium and the lower (outer) layers a consecutive solution method is proposed in the paper.

\section{References}

1 de Pater AD, Inleidend onderzoek naar het dynamisch gedrag van spoorstaven, PhD Thesis, Technische Hogeschool Delft, 1948, http:// books.google.hu/books?id=QsLYNwAACAA]

2 Zobory I, Zoller V, Zibolen E, Theoretical investigation into the dynamical properties of railway tracks using continuous beam model on elastic foundation, Periodica Polytechnica Transportation Engineering, 22(1), (1994), 3554.

3 Zobory I, Zoller V, Dynamic response of a railway track in case of a moving complex phasor excitation, Progress in Industrial Mathematics at ECMI 96, In: Brons M, Bendsoe MP, Soresen MP (eds.), Progress in Industrial Mathematics at ECMI 96, Teubner; Stuttgart, 1997, pp. 85-92, DOI 10.1007/978-3-322-96688-9_9

4 Zoller V, Zobory V, Relations between the motion-responses caused by fixed and moving loads acting on discretely supported strings and beams, Progress in Industrial Mathematics at ECMI 2000, In: Anile M, Capasso V, Greco A (eds.), Progress in Industrial Mathematics at ECMI 2000, Mathematics in Industry 1., Springer; Berlin, 2002, pp. 657-661. 